\title{
Measuring eHealth Literacy in Urban Hospitalized Patients: Implications for the Post-COVID World
}

\author{
Abbreviations \\ HL \\ BHLS \\ eHL \\ eHEALS \\ health literacy \\ Brief Health Literacy Screen \\ eHealth literacy \\ eHealth literacy scale
}

J Gen Intern Med 36(1):251-3

DOI: $10.1007 / \mathrm{s} 11606-020-06309-9$

(c) Society of General Internal Medicine 2020

\section{INTRODUCTION}

The COVID-19 pandemic has catalyzed use of technology in healthcare. ${ }^{1}$ This increased emphasis on technology requires adequate access and capability for optimal outcomes. Characterizing the current digital divide is essential to ensure novel technological advances do not exacerbate disparities. Hospitalization is a salient time to characterize the digital divide as inpatients face vulnerable transitions of care. eHealth interventions may be cost-efficient, efficacious ways to improve care transitions but only if patients can effectively use them.

We previously found that low health literacy (HL) was associated with increased need for help with online tasks among inpatients. ${ }^{2}$ However, it may be important to specifically measure digital "eHealth literacy" (eHL). ${ }^{3}$ In this study, we evaluated the eHL of a hospitalized, urban, predominantly black patient population.

\section{METHODS}

\section{Study Design}

This cross-sectional observational study was part of a larger quality-of-care study ${ }^{4}$ among adult inpatients on general medicine services. The University of Chicago Biological Sciences Division Institutional Review Board approved this protocol (\#IRB16-0763).

Prior Presentations This work was previously presented as an oral presentation at the regional Midwest SGIM Conference in Minneapolis, MN, in September 2019 and as a poster presentation at the International Conference on Communication in Healthcare in San Diego, CA, in October 2019.

Received September 9, 2020

Accepted October 8, 2020

Published online October 19, 2020

\section{Measures}

Research assistants consented participants, collected demographics, and administered the eHL (eHEALS), ${ }^{3}$ HL (Brief Health Literacy Screen), ${ }^{5}$ and technology survey (access and use) assessments.

\section{Data Analysis}

Descriptive statistics included medians, interquartile ranges (IQRs), and proportions. Continuous and categorical comparisons utilized Wilcoxon rank sums and chi-squared tests, respectively. Multivariable logistic regression analyses were conducted to determine differences in technology access/use by eHL level adjusting for HL, age, gender, race, and education. Spearman correlation coefficient was performed utilizing summative scores from the BHLS and eHEALS. $P<0.0036$ defined statistical significance by Bonferroni correction. All analyses used STATA version 15.1 (College Station, TX).

\section{RESULTS}

\section{Participant Characteristics}

Of 178 participants, the median age was 55 years, most were African American, half were female, and half completed some college or more. One-third had low HL and one-quarter had low eHL. The median eHEALS score was 30 (Fig. 1). The prevalence of low HL was similar between the low and moderate/high eHL groups (Table 1). No correlation was found between eHL and HL $(P=0.07)$.

\section{eHL and Technology Access and Use}

Most participants owned cellphones and smartphones without significant differences in ownership by eHL level. Significant differences existed in ownership of laptops and tablets by eHL level $(P<0.001$; Table 1). Fewer participants with low eHL (versus moderate/high eHL) reported having a texting plan $(P=0.007)$ or having home wireless Internet $(P<0.001$; Table 1). Low eHL scores were associated with less use of the Internet $(P<0.001)$. When examining health-related Internet use, we found that participants with low versus moderate/high eHL were less likely to report searching for health information online (33\% versus 78\%, $P<0.001$ ). Results were unchanged in all multivariable analyses (Table 1). 


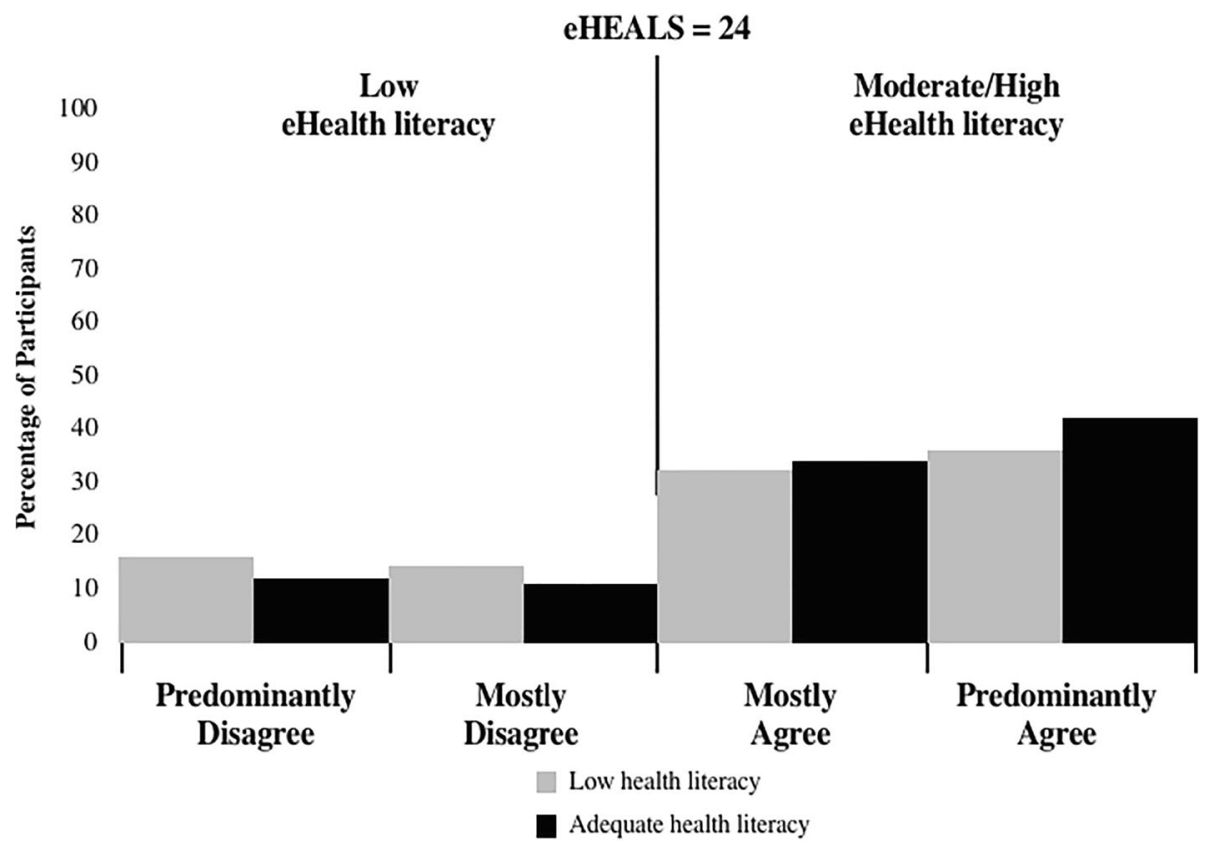

Figure 1 Histogram demonstrating eHEALS score distribution, stratified by health literacy (HL) level. eHEALS is an 8-item questionnaire with each item scored on Likert scale from 1 to 5 (from strongly disagree to strongly agree), with resultant composite score out of $40{ }^{14}$

Predominantly disagree scores range from 8 to 15.99 , mostly disagree scores range from 16 to 23.99 , mostly agree scores range from 24 to 31.99, and predominantly agree scores range from 32 to 40 . Low eHealth is defined by a score $<24$, moderate/high eHealth is defined by a score $\geq 24$. The median eHEALS score was 30 (IQR 11).

Table 1 Participant demographics; percentage of participants with technology access/use by eHL level with bivariate $P$ values; adjusted odds ratio and corresponding $P$ values for low eHealth literacy in multivariable analyses

\begin{tabular}{|c|c|c|c|c|c|c|}
\hline & $\begin{array}{l}\text { All participants }(n \\
=178)\end{array}$ & $\begin{array}{l}\text { Low eHL } \\
(n=46)\end{array}$ & $\begin{array}{l}\text { Mod/High } \\
\text { eHL }(n=132)\end{array}$ & $\begin{array}{l}\text { Bivariate } P \\
\text { value }\end{array}$ & $\begin{array}{l}\text { Multivariable AOR } \\
\text { (95\% CI)a }\end{array}$ & $\begin{array}{l}\text { Multivariable } P \\
\text { value }\end{array}$ \\
\hline \multicolumn{7}{|l|}{ Demographics } \\
\hline Age, median (IQR) & $55(26)$ & $60(23)$ & $52.5(24.5)$ & 0.002 & & \\
\hline Age $\geq 65 \%$ & $26 \%$ & $39 \%$ & $21 \%$ & 0.02 & & \\
\hline Female, $\%$ & $53 \%$ & $50 \%$ & $54 \%$ & 0.66 & & \\
\hline Race, \% & & & & 0.10 & & \\
\hline Black & $80 \%$ & $69 \%$ & $84 \%$ & & & \\
\hline White & $18 \%$ & $29 \%$ & $14 \%$ & & & \\
\hline Other & $2 \%$ & $2 \%$ & $2 \%$ & & & \\
\hline Some college or higher & $56 \%$ & $39 \%$ & $62 \%$ & 0.008 & & \\
\hline Low HL & $31 \%$ & $37 \%$ & $29 \%$ & 0.30 & & \\
\hline \multicolumn{7}{|l|}{ Technology access } \\
\hline Own cellphone & $90 \%$ & $85 \%$ & $92 \%$ & 0.18 & $0.7(0.2,2.1)$ & 0.50 \\
\hline Own smartphone & $80 \%$ & $68 \%$ & $84 \%$ & 0.02 & $0.2(0.08,0.6)$ & 0.004 \\
\hline Own laptop & $48 \%$ & $22 \%$ & $57 \%$ & $<0.001$ & $0.2(0.1,0.4)$ & 0.001 \\
\hline Own desktop & $42 \%$ & $28 \%$ & $46 \%$ & 0.03 & $0.4(0.2,0.8)$ & 0.01 \\
\hline Own tablet & $39 \%$ & $17 \%$ & $47 \%$ & 0.001 & $0.2(0.1,0.5)$ & 0.002 \\
\hline Have texting plan & $93 \%$ & $81 \%$ & $96 \%$ & 0.007 & $0.4(0.07,2.0)$ & 0.20 \\
\hline $\begin{array}{l}\text { Have data plan on } \\
\text { cellphone }\end{array}$ & $71 \%$ & $66 \%$ & $73 \%$ & 0.38 & $1.4(0.5,3.9)$ & 0.50 \\
\hline $\begin{array}{l}\text { Have unlimited data } \\
\text { plan }\end{array}$ & $63 \%$ & $53 \%$ & $66 \%$ & 0.16 & $0.7(0.3,1.9)$ & 0.50 \\
\hline $\begin{array}{l}\text { Have WiFi access at } \\
\text { home }\end{array}$ & $73 \%$ & $50 \%$ & $82 \%$ & $<0.001$ & $0.2(0.09,0.6)$ & 0.001 \\
\hline \multicolumn{7}{|l|}{ Technology use } \\
\hline Ever Internet use & $84 \%$ & $54 \%$ & $95 \%$ & $<0.001$ & $0.05(0.01,0.2)$ & $<0.001$ \\
\hline Daily Internet use & $66 \%$ & $37 \%$ & $76 \%$ & $<0.001$ & $0.2(0.09,0.6)$ & 0.003 \\
\hline Search for health & $67 \%$ & $33 \%$ & $78 \%$ & $<0.001$ & $0.1(0.05,0.3)$ & $<0.001$ \\
\hline \multicolumn{7}{|l|}{ information online } \\
\hline Download health apps & $32 \%$ & $17 \%$ & $36 \%$ & 0.05 & $0.4(0.1,1.4)$ & 0.16 \\
\hline $\begin{array}{l}\text { Post health information } \\
\text { online }\end{array}$ & $16 \%$ & $4 \%$ & $19 \%$ & 0.07 & $0.2(0.03,2.1)$ & 0.20 \\
\hline
\end{tabular}

eHL, eHealth literacy; Mod, moderate; IQR, interquartile range; HL, health literacy; significance level $P<0.0036$ by Bonferroni correction ${ }^{a}$ AOR is the best fit adjusted odds ratio for low eHealth literacy (eHL); low eHL is a binary variable with reference being moderate/high eHealth literacy; adjusted for: health literacy (binary), age (continuous), gender (binary), race (white, non-Hispanic black, other), education (less than any college versus some college or more) 


\section{DISCUSSION}

We found that one-quarter of participants had low eHL and that traditional drivers of the digital divide, specifically education and age, continue to be relevant even as technology access becomes more prevalent. Therefore, it is critical to remain focused on equity, ensuring technological innovations are implemented in an inclusive manner for individuals without confidence in health-related technology.

Smartphones may be an ideal interface when designing interventions for hospital-to-home transitions. Since access to and use of the Internet were significantly different by eHL level, this remains an area for improvement. In general, the "digital divide" today is more of a spectrum of ownership, use, and capability, not just a binary question of access.

Our work raises the question of what can be done to improve eHL, for which few data exist. Studies among diverse populations are needed to understand if/how eHL can be increased. Additionally, healthcare organizations should approach patients with "universal eHL precaution,"6 meaning eHealth intervention design and implementation should be conducted with a presumption that all patients may have low eHL; however, this "one size fits all" approach is likely insufficient.

This single site study has limited generalizability. Additionally, patient assessments were self-reported. Future studies could utilize scales with more objective measures.

In conclusion, as hospitals move to implement more health technology, especially in the post-COVID era, assessments of both HL and eHL will be important to identify patients who may need additional education to benefit. Further, this work emphasizes that with increasing prevalence of technology, we must be vigilant in implementing technology-based care in a way that is approachable and accessible across literacy levels.

Acknowledgments: We would like to thank Mary Akel for her assistance in preparing the manuscript for submission. We would also like to thank Ashley Hull, Eddie Kim, John Kim, Daniel Chang, and Brenda Munguia for their assistance in collecting the survey data.

Hanna Vollbrecht, $M D^{1}$

Vineet $M$. Arora, $M D^{2}$

Sebastian Otero, $B A^{2}$

Kyle A. Carey, $\mathrm{MPH}^{3}$

David O. Meltzer, $M D^{3}$

Valerie G. Press, $M D^{2}$
${ }^{1}$ Pritzker School of Medicine, University of Chicago, Chicago, IL, USA

${ }^{2}$ General Internal Medicine, Department of Medicine, University of Chicago,

Chicago, IL, USA

${ }^{3}$ Hospital Medicine, Department of Medicine, University of Chicago,

Chicago, IL, USA

Corresponding Author: Valerie G. Press, MD; General Internal Medicine, Department of Medicine, University of Chicago, Chicago, IL, USA (e-mail: vpress@bsd.uchicago.edu).

Funding This project was funded in part by an NIH NHLBI K23 (HL 118151), an American Lung Foundation Social Behavioral Award, and a Chicago Center for Diabetes Translation Research Pilot and Feasibility Grant. An NIH NIA grant \#4T35AG029795-10 provided support to Hanna Vollbrecht. Dr. Arora receives funding from NHLBI K24HL136859.

\section{Compliance with Ethical Standards:}

Conflict of Interest: Dr. Press reports previously consulting for Round Glass and currently serving as a subject matter expert for Vizient and as a consultant for Humana. The other authors have no conflicts to report.

\section{REFERENCES}

1. Rodriguez JA, Clark CR, Bates DW. Digital Health Equity as a Necessity in the 21st Century Cures Act Era. JAMA 2020. https://doi.org/10.1001/ jama.2020.7858

2. Vollbrecht H, Arora V, Otero S, Carey K, Meltzer D, Press VG. Evaluating the Need to Address Digital Literacy Among Hospitalized Patients: Cross-Sectional Observational Study. J Med Internet Res. 2020;22(6):e17519. https://doi.org/10.2196/17519

3. Norman CD, Skinner HA. eHEALS: The eHealth Literacy Scale. J Med Internet Res. 2006;8(4). https://doi.org/10.2196/jmir.8.4.e27

4. Meltzer D, Manning WG, Morrison J, et al. Effects of physician experience on costs and outcomes on an academic general medicine service: results of a trial of hospitalists. Ann Intern Med. 2002; 137(11):866-874.

5. Chew LD, Griffin JM, Partin MR, et al. Validation of Screening Questions for Limited Health Literacy in a Large VA Outpatient Population. J Gen Intern Med. 2008;23(5):561-566. https://doi.org/10.1007/s11606-0080520-5

6. Smith B, Magnani JW. New technologies, new disparities: The intersection of electronic health and digital health literacy. Int J Cardiol. 2019;292:280282. https://doi.org/10.1016/j.ijcard.2019.05.066

Publisher's Note: Springer Nature remains neutral with regard to jurisdictional claims in published maps and institutional affiliations. 